\title{
Antimicrobial Resistance Pattern of Bacterial Isolates among Nosocomial Infections (Urinary Tract Infection and Blood Stream Infection) from the Intensive Care Unit of a Tertiary Care Government Hospital in India
}

\author{
Manotosh Sutradhar ${ }^{1}$, Hirak Jyoti Raj ${ }^{2}$, Puja Trigunait ${ }^{3}$, Subinay Chhaule ${ }^{4}$, \\ Raja Ray ${ }^{5}$, Ujjaini Roy ${ }^{6}$, Rina Das ${ }^{7}$, Asutosh Ghosh ${ }^{8}$
}

\begin{abstract}
${ }^{1}$ Department of Critical Care Medicine, JPGME\&R, Kolkata, West Bengal, India. ${ }^{2}$ Department of Microbiology, JPGME\&R, Kolkata, West Bengal, India. ${ }^{3}$ Department of Critical Care Medicine, JPGME\&R, Kolkata, West Bengal, India. ${ }^{4}$ Department of Critical Care Medicine, JPGME\&R, Kolkata, West Bengal, India. ${ }^{5}$ Department of Microbiology, JPGME\&R, Kolkata, West Bengal, India. ${ }^{6}$ Department of Microbiology, JPGME\&R, Kolkata, West Bengal, India. ${ }^{7}$ Department of Forensic Medicine, R. G. Kar Medical College, Kolkata, West Bengal, India. ${ }^{8}$ Department of Critical Care Medicine, JPGME\&R, Kolkata, West Bengal, India.
\end{abstract}

\section{ABSTRACT}

\section{BACKGROUND}

Development of antimicrobial resistance (AMR) to different generations of common antibiotics by various organisms is rising alarmingly. A nationwide standard antibiotic policy is needed. We wanted to study the resistant organisms, the drug class to which organisms are becoming resistant and identify factors favoring the development of AMR for formulating an effective antibiotic policy.

\section{METHODS}

We conducted this observational study from January 2018 to May 2019 in a tertiary care hospital in India. Sample (urine, blood from ICU patients) processing, organism identification and antibiotic susceptibility tests were carried out as per the institutional guidelines in the Microbiology Department from where data collection was done.

\section{RESULTS}

Out of 500 samples, 145 (29\%) showed significant growth of organisms exhibiting resistance to either single or multiple drugs. Acinetobacter spp. was the most common organism isolated with a total of $40(27.58 \%)$, followed by Klebsiella spp. 23 (15.83\%), MRSA 20 (13.79\%), E. coli 15 (10.34\%), Pseudomonas 12 (8.27\%), Enterococci 12 (8.27\%), and CoNS 7 (4.82\%). Among GNB, Klebsiella and E. coli showed minimal resistance to polymyxins, fosfomycin, minocycline and tigecycline. Among Staphylococcus spp. maximum sensitivity was seen to teicoplanin, tobramycin, tigecycline and minocycline. Acinetobacter spp. showed high sensitivity to polymyxins, tobramycin, tetracycline, tigecycline and minocycline.

\section{CONCLUSIONS}

AMR was highly prevalent with hospital acquired organisms, and against commonly used antibiotics. Variation of resistance and sensitivity pattern with time and local microflora necessitates periodic AMR monitoring and rotation of antibiotics is suggested to restrict further emergence of resistance. Focusing on the organisms causing UTI and BSI and their resistance pattern, helps in selecting proper antibiotic therapy and in strengthening general sepsis measures.

\section{KEY WORDS}

Antibiotics, Microorganism, Nosocomial Infection, Antimicrobial Resistance, Observational Study
Corresponding Author:

Dr. Rina Das,

\#56A/34B,

P. Road, Kolkata-700041

West Bengal, India.

E-mail: drrinadas@rediffmail.com

\section{DOI: $10.14260 /$ jemds/2020/495}

How to Cite This Article:

Sutradhar M, Raj HJ, Trigunait P, et al. Antimicrobial resistance pattern of bacterial isolates among nosocomial infections (urinary tract infection and blood stream infection) from the intensive care unit of a tertiary care government hospital in India. J. Evolution Med. Dent. Sci. 2020;9(32):2284-2288, DOI: $10.14260 /$ jemds $/ 2020 / 495$

Submission 05-04-2020, Peer Review 30-06-2020, Acceptance 06-07-2020,

Published 10-08-2020.

Copyright (C) 2020 JEMDS. This is an open access article distributed under Creative Commons Attribution License [Attribution 4.0 International (CC BY 4.0)] 


\section{BACKGROUND}

Development of resistance against antibiotics in microorganisms has become a nightmare for the clinicians these days. Nosocomial infections are among the most serious infections acquired by ICU patients.[1] Antimicrobial resistance (AMR) is an emerging clinical problem in intensive care units (ICUs). Both AM-resistant Gram (-) negative bacilli (GNB) and Gram-positive bacteria (GPB) are reported as important causes of hospital-acquired infections. [1] Most of the Acinetobacter are Multi Drug resistant. Others are methicillin- resistant Staphylococcus aureus (MRSA), Vancomycin-resistant Enterococcus (VRE), Clostridium difficile, extended-spectrum $\beta$-lactamase-producing GNB, and Candida AMR is a growing health concern of not only India but the whole world.[2,3] Controlling infections in developing countries like India where infectious diseases still cause high morbidity and mortality, is a herculean task. ${ }^{[3,4]}$

Beside several intrinsic factors like gene amplification, point mutation etc, social factors like demographic variability, improper hygienic practices and population explosion have been enumerated for the emergence of the multidrug resistant (MDR) Escherichia coli by a study carried out in rural Tamil Nadu. ${ }^{[3,5]}$ Inappropriate and irrational uses of antibiotics in humans and animals for therapeutic and non-therapeutic use have been attributed as main causes for the emergence of community acquired and Nosocomial infections by World Health Organization (WHO).[6,7] Three clinically available $\beta$ lactamase inhibitor that can be combined with $\beta$-lactamase to reduce hydrolysis, are effective against class-A $\beta$-lactamases only and not against class B, C, D lactamases. Other class Bcarbapenemase inhibitors, which can be effective against carbapenemase producing organisms was under study, ${ }^{[8]}$ but most of the carbapenemase producing organisms are now a days sensitive to polymyxins. Increase in susceptibility to antibiotics by previously resistant gram negative organisms by following an antibiotic policy and by antibiotic rotation has been demonstrated in a study done on ventilator associated pneumonia among ICU patients who were started on antibiotics empirically by Didier Gruson et al.[9]

Due to less known centrally monitored data regarding AMR scenario in India and the geographical and time bound variations in antibiotic resistance and sensitivity that have been reported by many studies, our study was carried out with the following objectives

1. To identify the group of organisms developing resistance in BSI and UTI.

2. To assess the factors favourable for AMR development.

3. To address the local pathogens, thus to formulate empiric antimicrobial therapy.

4. To identify the classes of drugs, against which resistance has emerged.

5. To develop further policy or strengthen existing policy of general sepsis measures.

\section{METHODS}

This observational study was conducted from January 2018 to May 2019 in a tertiary care government hospital in India. Institutional ethical committee approval for the study was duly obtained (approval letter no-, dated-). Written informed consent was obtained from every study subject prior to data collection. Sample processing, identification of organisms to the genus/species level and antimicrobial sensitivity were done as per the Institutional guidelines for Clinical and Laboratory standards. Culture and sensitivity data of the organisms isolated from 500 non-repetitive samples of urine and blood from in patients (IP) of ICU were collected from the records of the Microbiology Department. Being an observational study, we did not go for a formal sample size calculation, however the sample size that we achieved is more than sufficient to answer the question as to what proportion of sample cases would turn out to be culture confirmed cases. Sample size calculation by raosoft sample size calculator [http://www.raosoft.com/samplesize.html] indicates that 377 sample will be required to answer this question with $5 \%$ margin of error and $95 \%$ confidence level assuming that the crude rate of culture confirmed cases would be $50 \%$.

Antibiotics tested for sensitivity against gram negative bacteria were $3^{\text {rd }} \& 4^{\text {th }}$ generation Cephalosporins, aminoglycosides, beta lactam and beta lactamase Inhibitor, Fluroquinolones, co-trimoxazole, carbapenems, tigecycline, tetracycline derivatives, polymyxins and fosfomycin. Antibiotic profiles for gram-positive bacteria were aminoglycosides, glycopeptides, fluroquinolones, penicillin derivatives, lincosamide, co-trimoxazole, macrolides, tetracycline derivatives and tigecycline. Organisms acquiring non-susceptibility to at least one agent in three or more antimicrobial categories were considered as MDR.

After getting an informed consent in English as well as in vernacular languages from the patients or relatives, a questionnaire to elicit their prior antibiotic history was undertaken. Other relevant data were supplemented from the patient's case sheet. All data were tabulated and analysed using descriptive statistics.

\section{Statistical Analysis}

Data have been summarized as counts and percentage and graphically represented as bar diagrams.

\section{RESULTS}

Out of 500 samples, 145 (29\%) showed significant growth of organisms and exhibited resistance to either single or multiple drugs. Remaining samples either had no organisms grown or had insignificant growth. Among the samples, 100 were from blood and 45 were urine samples [Tables-1]. Organisms isolated from various samples were Acinetobacter spp., E. coli, Klebsiella spp., Pseudomonas spp., Staphylococcus spp. (MRSA or CoNS), Enterococcus spp., Elizabethkingia meningoseptica, Citrobacter spp., Enterobacter spp., Myroids, Providentia rettgeri, Proteus mirabilis, Chryseobacterium indologenes etc. Acinetobacter spp. was the most common organism isolated with a total number of 40 (27.58\%). 36 (36\%) of Acinetobacter isolates were from blood and 4 were from urine. Klebsiella isolates were a total of 23 (15.86\%), 12 from urine and 11 from blood. 


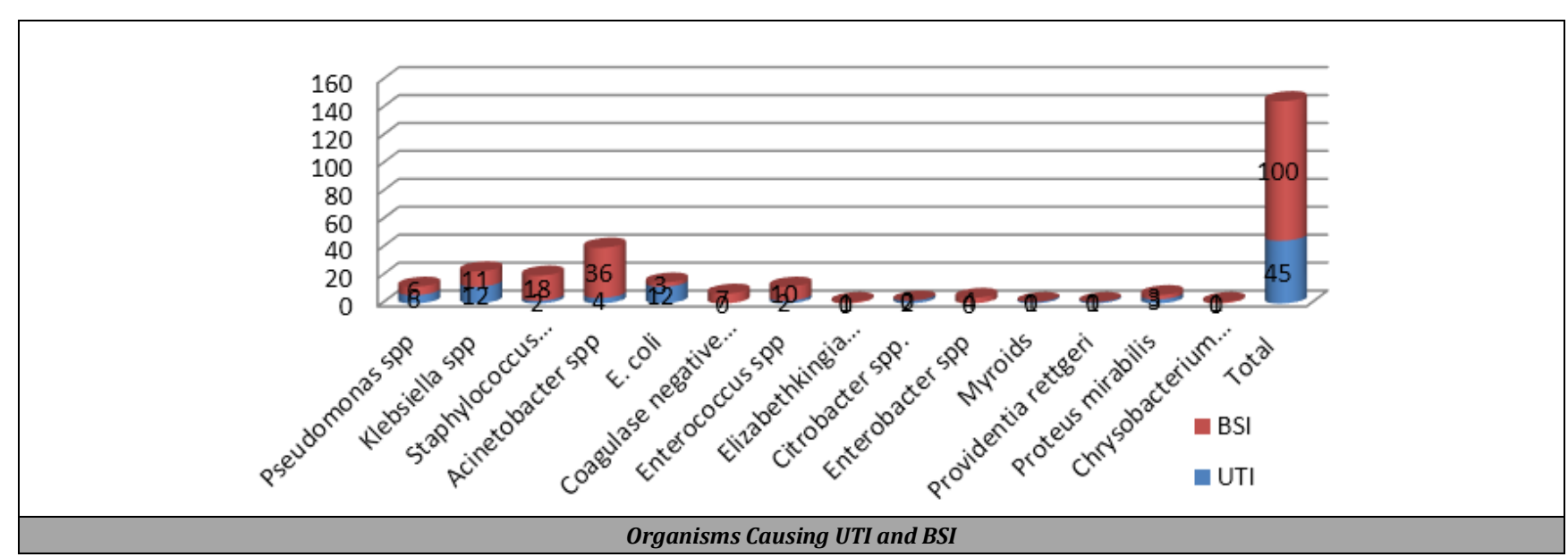

Among 15 (10.34\%) E. coli samples, 12 were from urine and 3 from blood. Amongst the 20 (13.79\%) MRSA isolates, 18 were from blood and 2 from urine samples. 6 (4.15\%) isolates of Pseudomonas were obtained from blood and urine samples each. 6 (4.13\%) Proteus were isolated, 3 each from urine and blood. 2 Enterococci were isolated from the urine samples and 10 from blood. Other positive samples found solely from blood were Coagulase negative staphylococci (CoNS) 7 (4.82\%), Enterobacter spp. 4 (2.75\%), Elizabethkingia meningoseptica 1, Chryseobacterium indologenes 1 . Some uncommon organisms isolated only from urine samples were Citrobacter spp.-2, Myroids-1, Providentia rettgeri-1

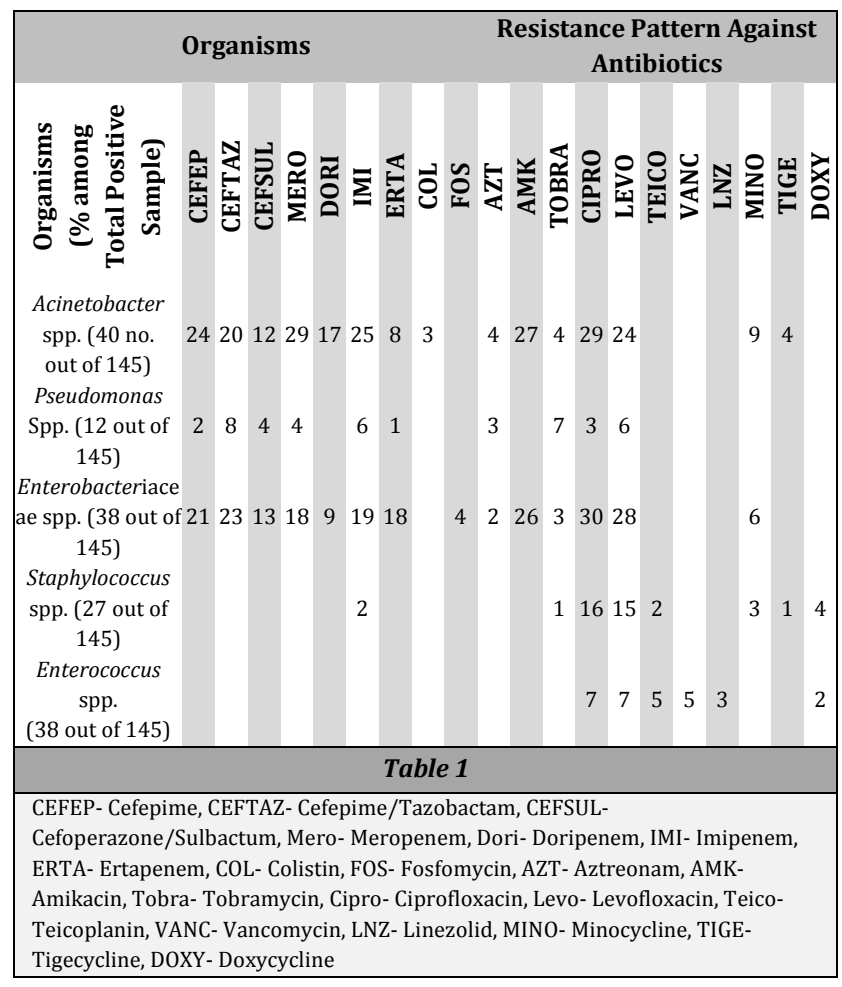

Among the commonly used antimicrobials against gram negative and gram-positive organisms in our study, maximum resistance was observed with co-trimoxazole followed by ampicillin, amoxicillin, amoxiclav, fluoroquinolones, third generation Cephalosporins and nalidixic acid. Least resistance or the highly sensitive antimicrobial to organisms isolated was observed with polymyxin $\mathrm{B}$, colistin, tigecycline, minocycline, amikacin, nitrofurantoin, gentamycin, clindamycin, doxycycline, macrolides and vancomycin [Figure 1].

Maximum resistance to multiple antimicrobials by organisms were observed by Acinetobacter spp. followed by Klebsiella, MRSA, E. coli and Pseudomonas [Figure 1].

Organisms from blood were the most resistant ones followed by those from urine samples. Sensitivity of isolated resistant organisms to various antimicrobials is depicted in Figure 2 to $5.41 \%$ of samples were collected before starting antimicrobials and $59 \%$ of the patients got antibiotics before sending samples. Culture report-based change in antimicrobials resulted good outcomes in many of the patients. Previous antimicrobial exposure history could not be elicited from more than $50 \%$ of subjects because of their unawareness. Patients unexposed to antibiotics was $10 \%$ and $30 \%$ were found having used antimicrobials before with or without completing the course.

\section{DISCUSSION}

Most of the positive culture growths were of GNBs. The gramnegative and few gram-positive (MRSA and CoNS) organisms isolated from ICU patients exhibited high antimicrobial resistance. This pattern is corroborative of the WHO community surveillance study report on pathogens suggesting prevalence of more resistant organisms among hospitalized patients than community acquired organisms.[10]

Acinetobacter spp., the most common and highly resistant organism in our study, was isolated mostly from blood than urine samples. Gram negative coccobacilli, Acinetobacter is a serious and emerging ICU infection. The organism has the ability to accumulate diverse mechanisms of resistance, leading to the emergence of strains resistant to all commercially available antibiotics.[11] Carbapenemresistant A. baumannii is a critical-priority pathogen on the WHO priority list of antibiotic-resistant bacteria for effective drug development.[12] Likewise, high percentage of ESBL producing E. coli was found in a study at a tertiary care hospital in Chennai.[13] More number of MDR E. coli was noted in our study, consistent with the results of many similar studies.[10,14,15] Highest resistance by E.coli was noted against penicillins followed by fluoroquinolones, co-trimoxazole, third generation cephalosporins and nalidixic acid in our study similar to many studies.[10,13,14,16] 
MRSA are highly prevalent in hospitalized post-surgical and trauma patients.[17,18,19,20] Hospital acquired MRSA is more virulent and MDR than community acquired one.[17,19] MRSA showed high resistance to co-trimoxazole, ampicillin/ amoxicillin and fluoroquinolones in our study. Least resistance was against macrolides, clindamycin and gentamycin. Vancomycin and amikacin were $100 \%$ effective on MRSA similar to other studies.[14,18,21,22-23]

MDR Pseudomonas was isolated in 12 out of 145 positive samples confirming it to be a common ICU infection as evidenced by many studies conducted at different parts of India. $[15,27,28]$ Sensitivity was observed more to cefepime and ertapenem than to fluoroquinolones in our study [Figure 1].

In our study, Klebsiella, like E. coli was found to be resistant to penicillins, co-trimoxazole, third generation cephalosporins, fluoroquinolones, nitrofurantoin. Least resistance was exhibited to tobramycin, gentamycin, amikacin mimicking the results of many studies.[24-26] Proteus showed variable sensitivity to amikacin, gentamycin and fluoroquinolones compared to $100 \%$ sensitivity reported by Shashi Chopra et al. among ICU patients in his study.[28] Enterococci was isolated from 2 urine and 10 blood samples and showed sensitivity to doxycycline, linezolid, moxifloxacin etc. Incidence of $28 \%$ isolation from ICU patients at Jalandhar, Punjab, was reported with similar sensitivity.[26]

Citrobacter was isolated from 2 urine samples, being resistant to nitrofurantoin and penicillin but sensitive to third generation cephalosporins, co-trimoxazole and fluoroquinolones in our study. This increased use of Quinolones might have resulted in increased resistance to them, which could have pushed down the use of Quinolones and increased that of third generation cephalosporins, increasing incidence of resistance to third generation cephalosporins, found in our study probably due to the increasing emergence of ESBL producing organisms following increased usage. Less resistance was noted against drugs such as gentamycin and amikacin. Restricted use of these drugs because of the cost and the need for assistance for parenteral administration may be the contributing factor for remaining sensitive still.

Empirical use of antibiotics in the community and in hospitalized patients has been highlighted in some studies.[27,28] Inappropriate use of antibiotics being an important contributing factor for the development of resistance has already been established. However, in a study among primary school children in rural Tamil Nadu, recent history of medication use was not identified as a significant risk factor for the emergence of MDR organisms. [5]

The lack of knowledge about the consequences of inappropriate use of antibiotics by the patients has been identified as an important cause for the emergence of AMR. Development of resistance was more by GNBs than by grampositive ones.

\section{CONCLUSIONS}

In this observational study, MDR organisms (gram negative, gram positive, and some rare species like Citrobacter) were isolated in 145 samples of urine and blood. They were likely due to nosocomial infections resistant to more than 2 - 3 groups of antibiotics. Geographical and time-based variation was clearly observed. However, emergence of AMR to prior antibiotic exposure could not be correlated. The changing pattern of resistant organisms could be identified though analysis from multiple centers. A proper antibiotic policy and strict follow up of guidelines along with antibiotic rotation and possible screening of health care workers and disinfecting them will help in controlling infections better.

Financial or Other Competing Interests: None.

\section{REFERENCES}

[1] Kollef MH, Fraser VJ. Antibiotic resistance in intensive care unit setting. Ann Intern Med 2001;134(4):298-314.

[2] Safdar N, Maki DG. The commonality of risk factors for nosocomial colonization and infection with antimicrobial resistant Staphylococcus aureus, Enterococcus, gram negative bacilli, clostridium difficile and Candida. Ann Intern Med 2002;136(11):834-44.

[3] Raghunath D. Emerging antibiotic resistance in bacteria with special reference to India. J Biosci 2008;33(4):593603.

[4] Gupta SK, Gupta P, Sharma P, et al. Emerging and reemerging infectious diseases future challenges and Strategy. J Clin Diagn Res 2012;6(6):1095-100.

[5] Seidman JC, Anitha KP, Kanungo R, et al. Risk factors for antibiotic-resistant $E$. coli in children in a rural area. Epidemiol Infect 2009;137(6):879-88.

[6] Sahoo KC, Tamhankar AJ, Sahoo S, et al. Geographical variation in antibiotic-resistant Escherichia coli isolates from stool, cow-dung and drinking water. Int J Environ Res Public Health 2012;9(3):746-59.

[7] Kulkarni SV, Narayan A, Indumathi VA, et al. Salmonella Paratyphi A in India-changing trends in presentation and antibiotic susceptibility. Asian J Med Sci 2011;2:14-7.

[8] Lee JH, Bae IK, Lee SH. New definitions of extendedspectrum $\beta$-lactamase conferring worldwide emerging antibiotic resistance. Med Res Rev 2012;32(1):216-32.

[9] Gruson D, Hilbert G, Vargas F, et al. Rotation and restricted use of antibiotics in a medical intensive care unit. Impact on the incidence of ventilator-associated pneumonia caused by antibiotic-resistant gram-negative bacteria. Am J Respir Crit Care Med 2000;162(3 Pt 1):837-43.

[10] World Health Organization. Community-based surveillance of antimicrobial use and resistance in resource-constrained settings: report on five pilot projects. WHO/EMP/MAR/ 2009.

[11] Lolans K, Rice TW, Munoz-Price LS, et al. Multicity outbreak of carbapenem-resistant Acinetobacter baumannii isolates producing the carbapenemase OXA40. Antimicrob Agents Chemother 2006;50(9):2941-5.

[12] Tacconelli E, Carrara E, Savoldi A, et al. Discovery, research, and development of new antibiotics: the WHO priority list of antibiotic-resistant bacteria and tuberculosis. Lancet Infect Dis 2018;18(3):318-27.

[13] Narayanaswamy A, Mallika M. Prevalence and susceptibility of extended spectrum beta-lactamases in urinary isolates of Escherichia coli in a tertiary care hospital, Chennai-South India. Internet J Med Update 2011;6(1):39-43. 
[14] Ganguly NK, Wattal C, Chandy SJ, et al. National Working Group Situation analysis: antibiotic use and resistance in India. Global Antibiotic Resistance Partnership-India March 2011.

[15] Peripi SB, Thadepalli VGR, Khagga M, et al. Profile of antibiotic consumption, sensitivity and resistance in an urban area of Andhra Pradesh, India. Singapore Med J 2012;53(4):268-72.

[16] Umadevi S, Kandhakumari G, Joseph NM, Kumar S, Easow JM, Stephen S, et al. Prevalence and antimicrobial susceptibility pattern of ESBL producing gram negative bacilli. J Clin Diagn Res 2011;5:236-9.

[17] Mohankumar A, Tamil Selvi S. Antibiotic pattern of methicillin resistant Staphylococcus aureus isolated from chronic wound of fisherman community. Int J Microbiol Res 2012;3:109-16.

[18] Goud RN, Agarval D, Nadagoudar PH, addad SM. Antibiotic sensitivity pattern of community-associated methicillinresistant Staphylococcus aureus (CA-MRSA) in High Schools, Bangalore city, Karnataka, South India. Int Med J Stud Res 2011;1:27-35.

[19] Rajaduraipandi K, Mani KR, Panneerselvam K, et al. Prevalence and antimicrobial susceptibility pattern of methicillin resistant Staphylococcus aureus: a multicentre study. Indian J Med Microbiol 2006;24(1):34-8.

[20] Tiwari HK, Das AK, Sapkota D, et al. Methicillin resistant Staphylococcus aureus: prevalence and antibiogram in a tertiary care hospital in western Nepal. J Infect Dev Ctries 2009;3(9):681-4.
[21] Kumar S, Joseph NM, Easow JM. Prevalence and current antibiogram of staphylococci isolated from various clinical specimens in a tertiary care hospital in Pondicherry. Internet J Microbiol 2012;10(1).

[22] Mahmood $\mathrm{K}$, Tahir $\mathrm{T}$, Jameel $\mathrm{T}$, et al. Incidence of methicillin-resistant Staphylococcus aureus (MRSA) causing nosocomial infection in a tertiary care hospital. Annals 2010;16(2):91-6.

[23] Saravanan M, Nanda A. Incidence of methicillin resistant Staphylococcus aureus (MRSA) from septicemia suspected children. Indian J Sci Technol 2009;2(12):36-9.

[24] Kousalya K, Thirumurugu S, Arumainayagam DC, et al. Antimicrobial resistance of bacterial agents of the upper respiratory tract in South Indian population. J Adv Pharm Technol Res 2010;1(2):207-15.

[25] Parveen RM, Khan MA, Menezes GA, et al. Extendedspectrum $\beta$-lactamase producing Klebsiella pneumonia from blood cultures in Puducherry, India. Indian J Med Res 2011;134(3):392-5.

[26] Chopra S, Mahajan G, Kaur J, et al. Septicemia due to MDR isolates in ICU: a therapeutic challenge. Asian J Pharm Health Sci 2012;2(4):476-80.

[27] Jamshidi M, Javadpour S, Eftekhari TE, et al. Antimicrobial resistance pattern among intensive care unit patients. Afr J Microbiol Res 2009;3:590-4.

[28] Shashikala, Kanungo R, Srinivasan S, et al. Emerging resistance to carbapenams in hospital acquired Pseudomonas infection: a cause for concern. Indian J Pharmacol 2006;38(4):287-8. 